\title{
Local Contrast based Texture features for Gliomas Grade Identification using Magnetic Resonance Images
}

\author{
Manu Gupta ${ }^{1}$, Shafee Vunnisa Sayyad ${ }^{2}$ \\ ${ }^{1}$ Depmartment of ECM, Sreenidhi Institute of Science \& Technology, Hyderabad, India, \\ Email: manugupta@sreenidhi.edu.in \\ ${ }^{2}$ Depmartment of ECE, Sreenidhi Institute of Science \& Technology, Hyderabad, India, \\ Email: shafeunnisa@ sreenidhi.edu.in
}

\begin{abstract}
Gliomas are most common brain tumor in children and adults worldwide and accounts for $80 \%$ of all malignant tumors. In this work, we proposed a novel method for glioma grade classification using texture feature set extracted from T2-weighted magnetic resonance images (MRI). Gray-level co-occurrence matrix (GLCM) parameters are computed from local Optimal Oriented Pattern (LOOP) transformed images to differentiate low grade and high grade glioma. Classification is carried out using support vector machine (SVM), Naive Bayes and k-nearest neighbor ( $\mathrm{k}-\mathrm{NN}$ ) classifier and their performance for glioma grade classification is accessed. SVM classifier outperforms other classifiers and achieved an accuracy of 95\%, sensitivity of $93 \%$ and specificity of $100 \%$ for classifying gliomas using proposed LOOP transformed based GLCM texture features.
\end{abstract}

Key words: Gliomas, Grade classification, Gray-level co-occurrence matrix (GLCM), Local Optimal Oriented Pattern (LOOP), Magnetic Resonance Imaging.

\section{INTRODUCTION}

Brain tumor caused due to abnormal growth of cells in brain, is one of the major causes of increased death rates in children and adults [1].As per the statistics from Central Brain Tumor Registry of the United States (CBTRUS) the 5-year survival rate for brain tumor is only $34 \%$ and $36 \%$ approximately for men and women respectively [2].

The most common types of primary brain tumors are gliomas. They are a heterogeneous group of diseases that vary from highly malignant and rapidly growing high grade tumor to historically benign and slowly growing low grade tumors. Gliomas account for about $28 \%$ of all brain tumors and $80 \%$ of all malignant tumors [2]. In children, gliomas account for almost $50 \%$ of all cancers in the central nervous system (CNS)[3].
Magnetic resonance imaging (MRI) and computed tomography (CT) are some of the imaging modalities used for diagnosis of brain tumor. Among other modalities, MRI is most frequently used as it provides vast information for tumor diagnosis based on its variable contrast property [4].

However, qualitative evaluation using MR images is difficult and time consuming process as large number of slices is produced. Also, diagnosis of tumors made based only on visual interpretation is prone to errors, as tumors vary widely in shape and appearance. Hence, various techniques for diagnosis and classification of brain tumor have been developed [4,5]. In most of the works, texture features have been extracted for tumor diagnosis using MR images. Texture features can be extracted by applying statistical and spectral approaches [6]. Statistical approach takes into consideration distributions and relationships between the gray levels of pixels in an image for extracting features from MR image. Kharrat et al. [7] used Gray-level co-occurrence matrix (GLCM) parameters to classify normal and abnormal MR images. Further, the classification of images in this study is performed using Support vector machine (SVM) classifier. Gupta et al.[8] presented a tumor classification model using texture features like mean, homogeneity, contrast etc. and moments based features to identify abnormality in MR image. Vani et al. [9] developed a system based on Discrete Wavelet Transform (DWT) based features for brain tumor detection. Classification of extracted features is then carried out using SVM classifier for differentiating normal and abnormal MR images. Hsieh et al. [10] also proposed an approach using texture features comprising of moment based global statistical features and GLCM based local statistical features for classification of gliomas. These techniques have a major drawback that they are sensitive to grayscale variations and rotations in input image.

In this study a texture feature set based on Local Optimal Oriented Pattern (LOOP) is developed for classification of gliomas. LOOP is used in this work to describe local spatial structure of an input image [11]. It has been proven to be invariant to illumination variations and rotations in input 
images. Statistical texture using GLCM based parameters [6] are computed from LOOP transformed MR images of glioma patients to measure intensity heterogeneity in them. Further, the grade classification of gliomas is carried out using various supervised classifiers and their performance for discriminating different grades is evaluated.

The remaining paper is organized as follows. In Section 2, the methodology followed for glioma grade identification in proposed method is described. This section describes the process of image preprocessing, tumor separation procedure, texture feature extraction methods and various classifiers used for glioma classification in proposed method. In section 3, the results obtained using proposed feature set are discussed. Also, the performance of the proposed method for glioma grade classification is compared with other recent works from literature in this section. Lastly, Section 4 concludes this paper.

\section{METHODS}

The methodology followed for grade identification of glioma in proposed work is shown in Figure 1. The process starts with collection of three dimensional (3D) volumes of brain tumor MRI. The images used in proposed work have been taken from the database of MICCAI 2012 Challenge [12]. In proposed work, T2-weighted 3D MRI volumes have been utilized for all the patients for glioma grade classification. The MR images obtained from the database are skull-stripped and have isotropic resolution of $1 \mathrm{~mm}$. Database also contains the ground truth for the tumor region. These ground truth images are used for tumor separation from MRI volumes and evaluation of classification results.

All the steps followed for glioma grade identification has been described in following sections.

\subsection{Preprocessing}

The brain MRI volumes are preprocessed for intensity inhomogeneity correction so as to improve the image quality. This process of reducing RF inhomogeneity in MR images is known as bias field correction. FMRIB Software Library (FSL) [13] is used in this study to perform bias field correction. After bias correction is performed, axial slices are extracted from 3D brain MRI volume and slices containing tumor are considered for subsequent analysis.

\subsection{Tumor Region Separation and Texture Feature Extraction}

The tumor region is separated from MRI axial slices by multiplying the T2-weighted MR images with the ground truth obtained from the database. The separated tumor region for one of the high grade and low grade glioma patient (in one of slices from complete MRI volume) is shown in Figure 2.
The texture features are then computed from these tumor separted T2-weighted MRI slices.

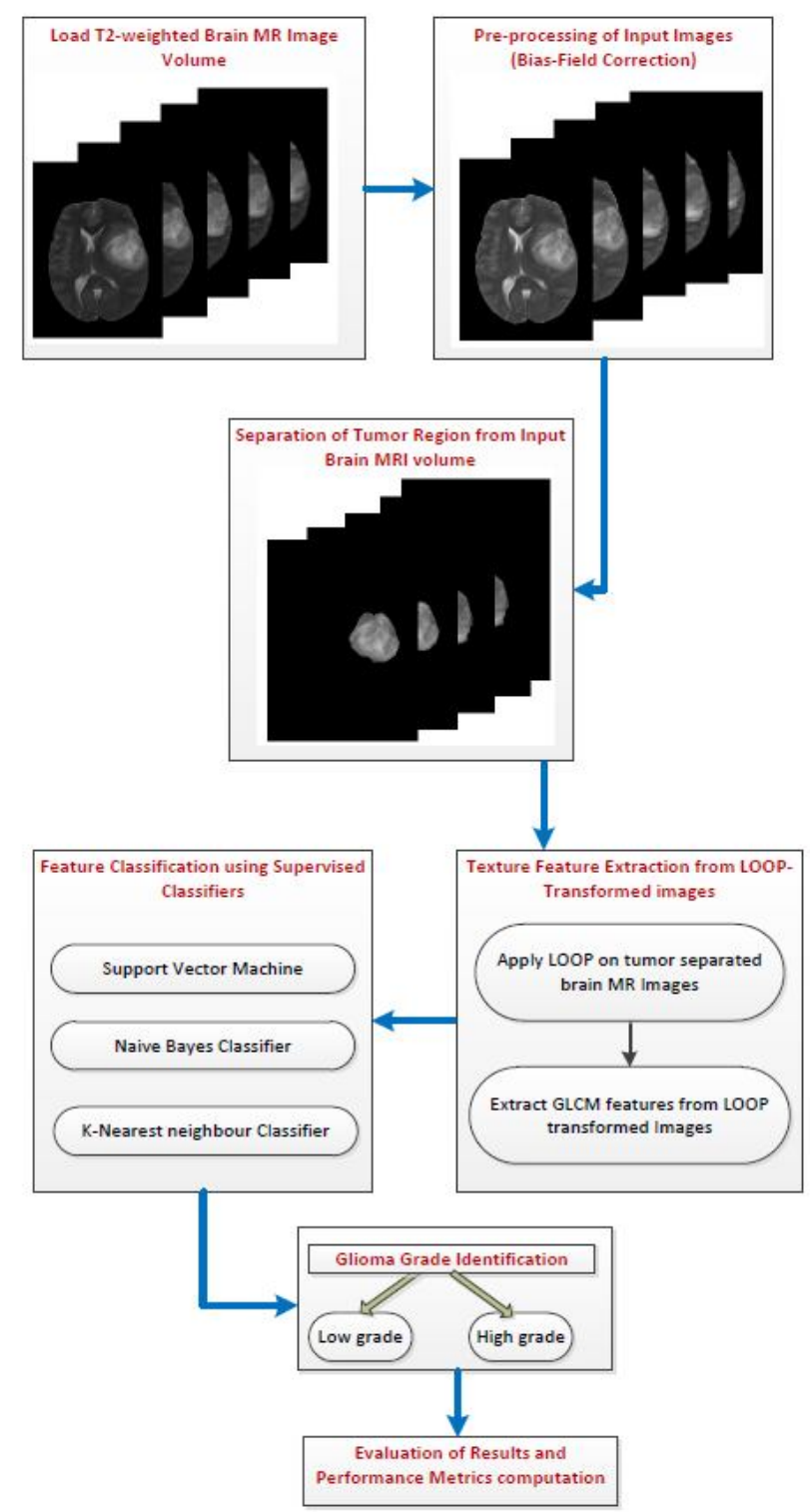

Figure 1: Flow diagram of proposed method for glioma grade classification

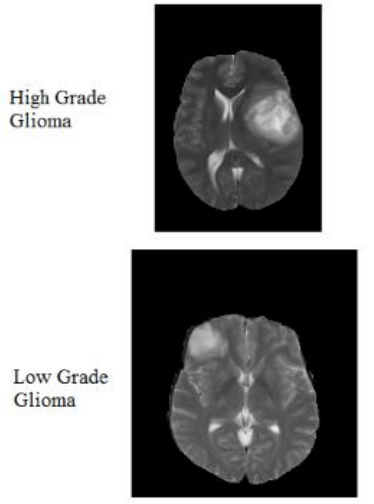

(a)
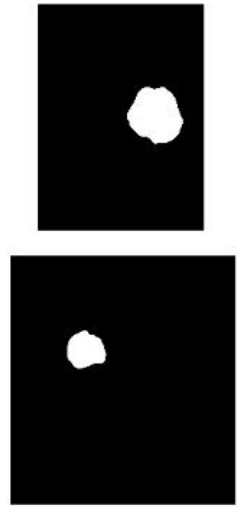

(b)
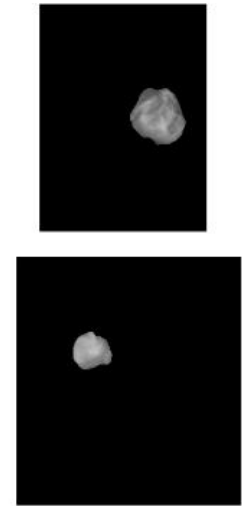

(c)
Figure 2: (a) Preprocessed T2-weighted image; (b) Ground truth; (c) Tumor region in T2-weighted image 
In this study an approach based on Local Optimal Oriented Pattern (LOOP) and Gray level co-occurrence matrix (GLCM) is presented. Firstly, the Local Optimal Oriented Pattern (LOOP) is applied on tumor separated MRI slices to generate LOOP transformed images. Next, the GLCM based second-order parameters are calculated from LOOP transformed images to extract texture information from MR Images of glioma patients.

\section{A. Local Optimal Oriented Pattern (LOOP) Generation}

LOOP descriptor is a local pattern descriptor which computes local contrast and orientation of image at various scales [11]. This descriptor is robust against rotation variance and local intensity variations in input image. The eight Kirsch masks are used in LOOP method corresponding to 8 directions [11] as displayed in Figure 3. These masks will give measure of strength of intensity variations in input image in these directions and are used to assign decimal-to-binary weightage while computing LOOP value for the pixel in an image.

$$
\begin{aligned}
& {\left[\begin{array}{ccc}
-3 & -3 & 5 \\
-3 & 0 & 5 \\
-3 & -3 & 5
\end{array}\right] \quad\left[\begin{array}{ccc}
-3 & 5 & 5 \\
-3 & 0 & 5 \\
-3 & -3 & -3
\end{array}\right] \quad\left[\begin{array}{ccc}
5 & 5 & 5 \\
-3 & 0 & -3 \\
-3 & -3 & -3
\end{array}\right] \quad\left[\begin{array}{ccc}
5 & 5 & -3 \\
5 & 0 & -3 \\
-3 & -3 & -3
\end{array}\right]} \\
& \text { East North East North North West } \\
& \begin{array}{c}
\begin{array}{ccc}
{\left[\begin{array}{ccc}
5 & -3 & -3 \\
5 & 0 & -3 \\
5 & -3 & -3
\end{array}\right]} \\
\text { West }
\end{array} \quad\left[\begin{array}{ccc}
-3 & -3 & -3 \\
5 & 0 & -3 \\
5 & 5 & -3
\end{array}\right] \\
\text { South West }
\end{array}
\end{aligned}
$$

Figure 3: Kirsch masks in eight directions

LOOP pattern is computed for the tumor separated regions obtained in previous step (refer Figure 2). Let the tumor separated T2-weighted image is represented as $\mathrm{I}_{\mathrm{T}}$ and intensity at pixel $(p, q)$ be $i_{\mathrm{c}}$. Then the LOOP transformed value for pixel $(p, q)$ for $3 \times 3$ neighborhood is given as follows:

$$
\operatorname{LOOP}(p, q)=\sum_{n=0}^{7} r\left(i_{n}-i_{c}\right) \cdot 2^{w_{n}}
$$

where, $i_{n}$ be intensity levels of the neighboring pixels and

$$
r(t)=\left\{\begin{array}{lr}
1 & \text { if } t \geq 0 \\
0 & \text { otherwise }
\end{array}\right.
$$

The weights are assigned using responses of Kirsch masks. In this process, corresponding to Kirsch mask in eight directions shown in Figure 3, eight directional responses are computed. These responses are given rank on basis on their magnitude. According to the rank of output of Kirsch masks the exponential weights $w_{n}$ are assigned in LOOP pattern computation. The LOOP code for all the pixels in input image are computed by repeating the same process along its rows and columns. The LOOP transformed image for one of the axial slices in MRI volume of glioma patient is shown in Figure 4.

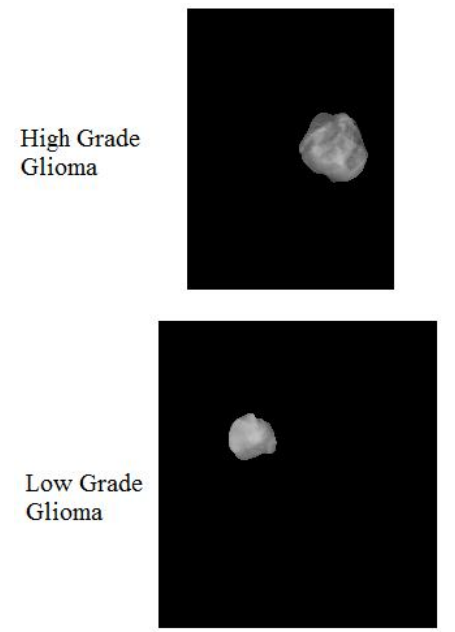

(a)
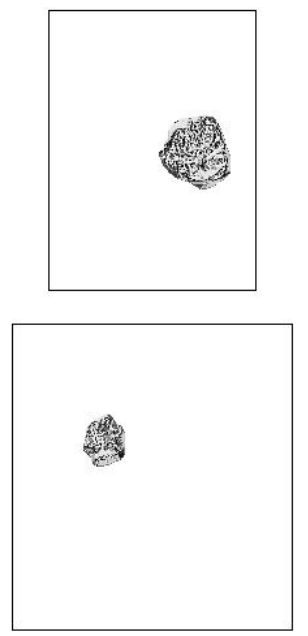

(b)
Figure 4: LOOP transformed images for one of the slices from MRI volume of HG and LG glioma patients considered in Figure 2.

\section{B. GLCM Texture Features Extraction}

Texture is one of the important characteristics which provide information about structural arrangement of surface [4]. In proposed study, texture variations in tumorous images are computed using second-order Gray level co-occurrence matrix (GLCM) based intensity features. These features are calculated for LOOP transformed images of tumor separated region in T2-weighted MR images (refer Figure 4).

GLCM method measures the probability of occurrence of each pixel pair in an image for the particular distance and angle [6]. It extracts information about spatial distribution of pixels in a specific neighborhood. In this study, the GLCM parameters are computed in four directions at angles of $0^{\circ}, 45^{\circ}, 90^{\circ}$ and $135^{\circ}$ with distance $\mathrm{d}=1$ and averaged to form a single feature set. Let the co-occurrence matrix be defined as $M=[m(i, j) \mid d, \theta]$ which gave frequency of occurrence pixel with gray level $i$ and its neighboring pixels with gray level $j$ at distance $d$ and angle $\theta$. From this matrix, the following second order GLCM features are computed $[6,10]$ :

$$
\begin{array}{ll}
\text { i. } & \text { Contrast }=\sum_{n} n^{2}\left\{\sum_{i} \sum_{j} m(i, j)\right\},|i-j|=n \\
\text { ii. } & \text { Correlation }=\frac{\sum_{i} \sum_{i}\left(i-\mu_{x}\right)\left(j-\mu_{y}\right) m(i, j)}{\sigma_{x} \sigma_{y}} \\
\text { iii. } & \text { Dissimilarity }=\sum_{i} \sum_{j} m(i, j)|i-j| \\
\text { iv. } & \text { Energy }=\sum_{i} \sum_{j} m(i, j)^{2} \\
\text { v. } & \text { Entropy }=-\sum_{i} \sum_{j} m(i, j) \log (m(i, j))
\end{array}
$$




$$
\begin{gathered}
\text { vi. } \quad \text { Homogeneity }=-\sum_{i} \sum_{j} \frac{1}{1+i-j} m(i, j) \\
\text { vii. } \quad \text { Difference Variance }=\sum_{i} i^{2} m_{x-y}(i) \\
\text { viii. } \quad \text { Maximum Probability }=\max _{i, j} m(i, j) \\
\text { ix. } \quad \text { Sum Of Squares: Variance }= \\
\sum_{i} \sum_{j}(i-\mu)^{2} m(i, j)
\end{gathered}
$$

x. Difference Entropy =

$$
-\sum_{i} m_{x-y}(i) \log \left(m_{x-y}(i)\right)
$$

where $\mu_{x}, \mu_{y}$ and $\sigma_{x}, \sigma_{y}$ are mean and standard deviation of probability matrix along rows $\left(m_{x}\right)$ and columns $\left(m_{y}\right)$.

The proposed feature set, consisting of LOOP transformed based GLCM texture features are computed for all the glioma patients using T2-weighted MR Images. After feature extraction the classification is performed using various supervised classifiers to classify the given brain tumor image as LG or HG.

\subsection{Classification}

In this work, we have studied and compared the performance of three most frequently used supervised classifiers in previous works: SVM, K-Nearest Neighbor (k-NN) and Naive Bayes classifier $[4,7,9,14,15]$ for classification of glioma images using extracted feature set.

In SVM classifier the input data is separated in two classes by identifying an optimal hyper-plane which distinguishes data points from one class to another. The decision rule for separating the classes was obtained from the training set whose classes are known in prior. The hyperplane obtained from decision rule was then applied for identifying the class of data in the testing set [7]. SVM basically maps the input vectors into some high dimensional feature space using certain functions known as kernels. Linear function is used as a kernel in this study for classification of brain tumor images.

$\mathrm{k}$-nearest neighbor is a supervised learning algorithm where the class of a new test instance or a data is determined based on the class of majority of $\mathrm{k}$ closest data points in the training set [14]. As for the data points in training set the class is predefined, $\mathrm{k}$ nearest neighbor algorithm used neighborhood classification as the prediction value to classify an unknown test instance/data point.

Naive Bayes classifier is a simple probabilistic linear classifier based on Bayes theorem. It classified data into associated classes based on their attributes or features. The data set is assigned to a class which generates the maximum conditional posterior probability with available attributes as input using Bayes rule [15].
K-fold cross validation method is implemented to split the features extracted in the proposed model into training and testing set [16]. This method has an advantage that it makes full use of the limited sample dataset for classification so as to evaluate performance of proposed feature set for glioma grade identification. It also ascertains that for training and validation of classifier whole dataset is considered. The classifier's performance accuracy is computed by averaging the accuracy obtained across k-trials.

\section{RESULTS AND DISCUSSION}

In proposed study, the LOOP transformed based GLCM texture features are extracted from brain tumor images to determine the tumor grade and degree of malignancy (low grade or high grade). The extracted texture features quantify the intensity distribution in the separated tumor region and are invariant to intensity variations and rotations in the input image. The evaluation of proposed method for glioma grade classification is performed using various supervised classifier. Twenty glioma patients from MICCAI 2012 Challenge database are used to evaluate the performance of proposed method. The results obtained from proposed brain tumor diagnosis model are summarized in this section.

The algorithm for texture feature extraction and tumor grade classification in proposed study are developed and simulated in MATLAB 2013a. The performance evaluation metrics used for quantitative assessment of proposed brain tumor detection technique are described as follows:

- Accuracy - It gives the percentage of correct predictions to the total cases considered in given dataset.

$$
\text { Accuracy }=\frac{T P+T N}{T P+T N+F P+F N} \times 100
$$

- Sensitivity- It gives percentage of true positives to total number of positive cases for the given dataset.

$$
\text { Sensitivity }=\frac{T P}{(T P+F N)} \times 100
$$

- Specificity- It gives percentage of true negatives to total number of negative cases for the given dataset.

$$
\text { Specificity }=\frac{T N}{(T N+F P)} \times 100
$$

where, TP and FP refers to true positive and false positive, and FN and TN refers to false negative true negative cases respectively. In the proposed study, high grade tumors are considered positive cases whereas low grade tumors are referred as negative cases.

The results obtained for various performance metrics for different classifiers using k-fold cross validation technique (with $\mathrm{k}=5$ ) are 
summarized in Table I. It is observed that SVM classifier provides better results for glioma classification in comparison to Naive Bayes and $\mathrm{k}-\mathrm{NN}$ classifier.

Table 1: Performance comparison of various classifiers using proposed feature set for glioma classification

\begin{tabular}{llll}
\hline Classifier & $\begin{array}{l}\text { Accuracy } \\
\text { (in \%) }\end{array}$ & $\begin{array}{l}\text { Sensitivity } \\
\text { (in \%) }\end{array}$ & $\begin{array}{l}\text { Specificity } \\
\text { (in \%) }\end{array}$ \\
\hline $\begin{array}{l}\text { SVM } \\
\text { (linear kernel) }\end{array}$ & 95 & 93 & 100 \\
Naïve Bayes & 90 & 93 & 83 \\
k-NN & 90 & 93 & 83 \\
\hline
\end{tabular}

We have compared the performance of feature set extracted in this study with methods presented by Gupta et. al. [8] and Hsieh et al. [10] using SVM classifier. The specificity, sensitivity and accuracy values for the same are shown in Figure 5. The proposed method provides accuracy of $95 \%$, whereas for method presented in [8] and [10] the classification accuracy is $85 \%$ and $75 \%$ respectively. Also the sensitivity and specificity values for proposed method are higher as compared to other works from literature. The reason for this can be that the GLCM based texture features extracted from LOOP transformed images of separated tumor region in proposed study can better assess the texture differences in LG (benign) and HG (malignant) tumors. Also the characteristics of LOOP method provides robustness to intensity and rotation variations caused due to use of different MRI scanners and helps in improving classification efficiency.

The results obtained demonstrates that the features extracted for brain tumor diagnosis may efficiently classify low grade and high grade tumors and can help the radiologists to quantitatively assess brain tumors non-invasively for their diagnosis.

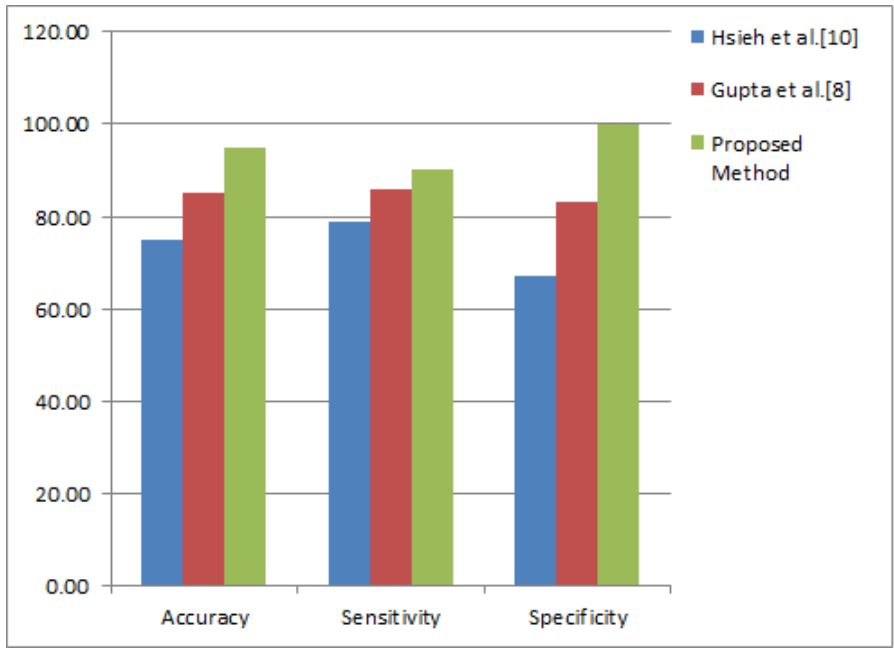

Figure 5: Performance comparison of different methods for glioma grade classification

\section{CONCLUSION}

The novel non-invasive quantitative feature set for brain tumor diagnosis comprising of texture features computed using LOOP and GLCM method is presented in this study. Brain MRI volumes of T2-weighted images were utilized in this study for extracting texture information from tumor regions and classify images as of LG or HG gliomas. The proposed model is giving significantly better results in comparison to other state of art method from literature. Use of rotation and intensity invariant LOOP transformed GLCM based texture feature set in proposed study improves the classification efficiency of our brain tumor diagnosis system. The proposed feature set can assist the radiologists and clinicians in non-invasive detection of brain tumor.

\section{ACKNOWLEDGEMENT}

The authors would thank Director, Sreenidhi Institute of Science and Technology, Hyderabad for providing research facilities.

\section{REFERENCES}

1. A. Munshi. Central nervous system tumors: Spotlight on India, South Asian journal of cancer, vol. 5, no. 3, pp. 146-147, 2016. https://doi.org/10.4103/2278-330X.187588

2. National Brain Tumor Society, Brain Tumors by the Numbers, 2016.

3. J.E. Minturn and M.J. Fisher. Gliomas in children, Current treatment options in neurology, vol. 15 , no. 3 , pp.316-327, 2013. https://doi.org/10.1007/s11940-013-0225-x

4. E.S. El-Dahshan, H.M. Mohsen, K. Revett, A.B. Salem. Computer-aided diagnosis of human brain tumor through MRI: A survey and a new algorithm, Expert systems with Applications, vol. 41, no. 11, pp. 5526-5545, 2014.

https://doi.org/10.1016/j.eswa.2014.01.021

5. K. Sudharani, A. Swapnarani, K. Manikumari, T.C. Sarma and K.Satyaprasad.. LABVIEW based brain tumor area and length Detection in CT and MRI scan images, in International Journal of Advanced Trends in Computer Science and Engineering, vol. 2, no. 5, pp. 70-74, 2013.

6. A. Materka, and M. Strzelecki. Texture analysis methods-a review, Technical university of Lodz, institute of electronics, COST B11 report, Brussels, pp. $1-33,1998$.

7. A. Kharrat, K. Gasmi, M. B. Messaoud, N. Benamrane, and M. Abid. A hybrid approach for automatic classification of brain MRI using genetic algorithm and support vector machine, Leonardo journal of sciences, vol. 17, no. 1, pp. 71-82, 2010.

8. N. Gupta and P. Khanna. A fast and efficient computer aided diagnostic system to detect tumor from brain magnetic resonance imaging, International Journal of 
Imaging Systems and Technology, vol. 25, no. 2, pp. 123-130, 2015. https://doi.org/10.1002/ima.22128

9. V. Vani and M. Kalaiselvi Geetha.. Implementation of Classification System for Brain Tumor using DWT, in International Journal of Advanced Trends in Computer Science and Engineering, vol. 5, no. 6, pp. 82-88, 2016.

10. K. L. C. Hsieh, C. M. Lo, and C. J. Hsiao. Computer-aided grading of gliomas based on local and global MRI features, Computer methods and programs in biomedicine, vol. 139, pp. 31-38, 2017. https://doi.org/10.1016/j.cmpb.2016.10.021

11. T. Chakraborti, B. McCane, , S. Mills and U. Pal. LOOP Descriptor: Local Optimal-Oriented Pattern, IEEE Signal Processing Letters, vol. 25, no. 5, pp. 635-639, 2018. https://doi.org/10.1109/LSP.2018.2817176

12. B.H Menze, A. Jakab, S. Bauer, J. Kalpathy-Cramer, K. Farahani, J. Kirby, Y. Burren, N. Porz, J. Slotboom, R. Wiest and L. Lanczi. The multimodal brain tumor image segmentation benchmark (BRATS), IEEE Transactions on Medical Imaging, vol. 34, no. 10, pp. 1993-2024, 2015.

13. S.M. Smith, M. Jenkinson, M.W. Woolrich, C.F. Beckmann, T.E. Behrens et al. Advances in functional and structural MR image analysis and implementation as FSL, Neuroimage, 23: S208-S19, 2004. https://doi.org/10.1016/j.neuroimage.2004.07.051

14. N. V. Chavan, B. Jadhav, and P. Patil. Detection and classification of brain tumors, International Journal of Computer Applications, vol. 112, no. 8, pp. 48-53, 2015.

15. Q. Ain, I. Mehmood, S.M. Naqi, and M.A. Jaffar. Bayesian Classification using DCT features for brain tumor detection," in Proc. International Conference on Knowledge-Based and Intelligent Information and Engineering Systems, Springer, 2010, vol. 6276, pp. 340-349.

https://doi.org/10.1007/978-3-642-15387-7_38

16. A.A. Hasseim, R. Sudirman and P.I. Khalid. Handwriting classification based on support vector machine with cross validation, Engineering, vol. 5, no. 5, pp. 84-87, 2013.

https://doi.org/10.4236/eng.2013.55B017 